\title{
Nanopore Detection of Single Molecule RNAP-DNA Transcription Complex
}

\author{
C. Raillon ${ }^{\dagger}$ P. Cousin, ${ }^{\ddagger}$ F. Traversi, ${ }^{\dagger}$ E. Garcia-Cordero, ${ }^{\dagger}$ N. Hernandez, ${ }^{\ddagger}$ and A. Radenovic ${ }^{*}{ }^{\dagger}$ \\ ${ }^{\dagger}$ Laboratory of Nanoscale Biology, Institute of Bioengineering, School of Engineering, EPFL, 1015 Lausanne, Switzerland \\ ${ }^{\ddagger}$ Center for Integrative Genomics, Faculty of Biology and Medicine, University of Lausanne, Lausanne, Switzerland
}

Supporting Information

ABSTRACT: In the past decade, a number of single-molecule methods have been developed with the aim of investigating single protein and nucleic acid interactions. For the first time we use solid-state nanopore sensing to detect a single $E$. coli RNAP-DNA transcription complex and single E. coli RNAP enzyme. On the basis of their specific conductance translocation signature, we can discriminate and identify between those two types of molecular translocations and translocations of bare DNA. This opens up a new perspectives for

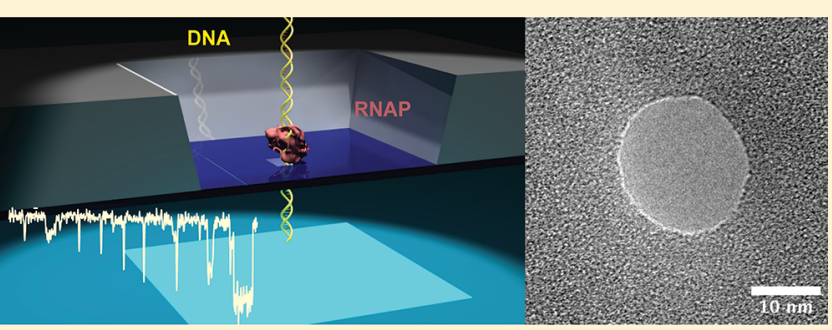
investigating transcription processes at the single-molecule level.

KEYWORDS: Solid-state nanopore, E. coli RNA polymerase, DNA, single-molecule, AFM, protein shape and dipole

$\mathrm{I}$ n only a decade since its invention, solid-state nanopore sensing $^{1}$ has emerged as a versatile method for singlemolecule manipulation and analysis. So far, solid-state sensing has been employed to detect and characterize single molecules of double- and single-stranded DNA, ${ }^{2-7}$ RNA, $^{7,8}$ and proteins. $^{9-12}$ However, only few studies have addressed the detection of DNA-binding molecules such as proteins ${ }^{10,13,1415}$ or small molecules. ${ }^{16-18}$ DNA-protein interactions can be classified as nonspecific or specific, and both types of interactions have been examined by nanopore sensing. As a prototypical nonspecific DNA binding protein, several groups ${ }^{10,13,1419,20}$ have investigated binding of recombination protein A (RecA) to DNA, which is known to form stable nucleoprotein filaments on dsDNA. By using solid-state nanopores, Kowalczyk et al. ${ }^{10}$ could detect as few as five RecA proteins bound to DNA and demonstrated high resolution of solid-state nanopores sensing, a property that had been mostly associated with biological pores. ${ }^{21}$ As for specific DNA binding proteins, Dorvel et al. ${ }^{15}$ studied the prototypical restriction endonuclease EcoRI which, like other enzymes that specifically recognize DNA sequences, finds its cognate site through a three-step process: nonspecific binding to DNA, linear diffusion along the strand until it encounters the target site, and binding to its restriction site with a very high affinity. They probed how strongly EcoRI binds to its specific target site by using pores smaller that the protein-DNA complex; however, they failed to detect the EcoRI-DNA complex. Here we show that solid-state nanopores can be used to probe and sense single proteins bound to DNA.

Processes such as replication, transcription, and translation require the information encoded in the sequence of nucleic acids to be read and copied by molecular machines in a directional manner. ${ }^{22}$ The molecular machines that carry out these processes have the property of moving along DNA and are thus termed translocases. In addition, they have the property of recognizing, with the help of accessory factors, specific DNA sequences corresponding to origins of replication, promoters, or translation start sites. These translocases are thus capable of adopting specific and nonspecific DNA binding modes as they transition from the initiation to the elongation step of their respective reactions. Here, we have used solid-state nanopores to distinguish a single protein bound to DNA from protein and DNA alone (Figure 1). We have chosen the E. coli RNA polymerase (RNAP), the enzyme that catalyzes the first step of gene expression, transcription, in which the information stored in DNA is copied into the mRNA (mRNA), ${ }^{23}$ as a model to study the DNA-protein interaction of a translocase paused during the elongation step. The advent of singlemolecule techniques have allowed to follow the individual transcription traces in real time and to reveal their stochastic nature $^{24-26}$ (alternation in periods of continuous translocation and pauses) together with the RNAP conformational changes ${ }^{27}$ that occur during the transcription process.

The experimental setup we used is shown in Figure 1. The $3 \mathrm{D}$ view illustrates an E. coli RNAP-DNA complex translocating through a nanopore. The plus and minus signs symbolize the $\mathrm{Ag} / \mathrm{AgCl}$ electrodes used to apply a potential across the nanopore. Since the DNA and the external surface of RNAP are negatively charged, the complex is attracted toward the positive electrode. When the molecule translocates through the nanopore, the base current is expected to be significantly reduced because the RNAP-DNA complex blocks the regular

Received: August 4, 2011

Revised: February 21, 2012

Published: February 28, 2012 


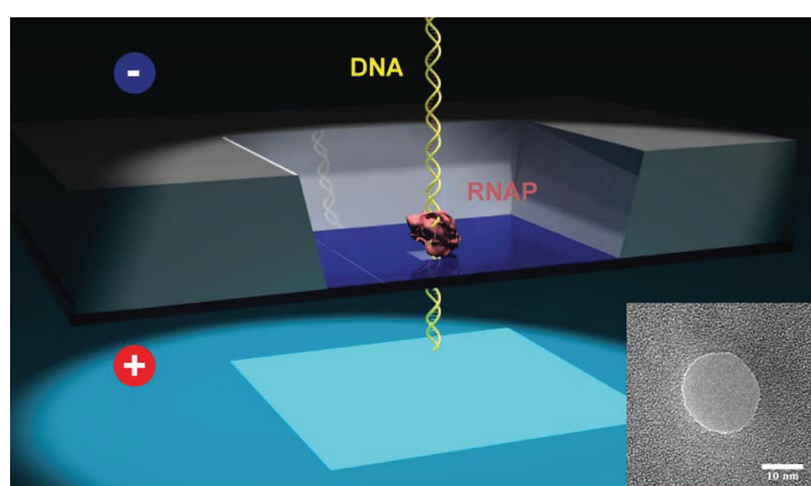

Figure 1. Schematic of the experimental setup (not to scale). An ionic current is carried by $\mathrm{K}^{+}$and $\mathrm{Cl}^{-}$ions flowing through a voltage-biased nanopore. When the RNAP-DNA transcription complex translocates through the nanopore, it partially blocks this flow of ions and is detected by our nanopore setup. Inset shows a typical $20 \mathrm{~nm}$ diameter pore used in such an experiment.

flow of ions going through the nanopore. A model of the holoenzyme E. coli RNAP bound to a 92 bp DNA fragment is displayed in Figure 2a. The schematic was drawn with the software UCSF Chimera based on the three-dimensional electron microscopy structure of E. coli RNAP obtained by Lawson et al. $^{28}$ (PDB reference 3IYD, resolution $1.98 \mathrm{~nm}$ ). The complex is oriented to show all its subunits, namely two $\alpha$, one $\beta$, and one $\beta^{\prime}$ subunit forming the core polymerase, as well as a $\sigma$ subunit which confers to the core polymerase the ability to bind specifically to promoter sequences. The ellipsoidal holoenzyme (the core enzyme with the sigma factor) with overall dimensions of $12 \times 15 \times 18 \mathrm{~nm}^{3}$ has a molecular weight of $\sim 480 \mathrm{kDa}$. The core enzyme is slightly smaller with overall dimensions of $11 \times 16 \times 16 \mathrm{~nm}^{3}$. Their net molecular charge and the dipole are computed using the Protein Dipole Moments Server. ${ }^{29}$ Only the subunits corresponding to the core and holoenzyme are used and the values found are respectively $-69 e$ and $-77 e$. The overall high net negative charge, even when a significant fraction is screened by the positively charged counterions, ${ }^{30}$ is likely to promote translocation events. Moreover, the dipole moment of E. coli RNAP might induce preferential orientation of the ellipsoidal molecule when translocating the nanopore.

Translocation of bare DNA through solid-state nanopores has been typically analyzed using scatter plots of translocation dwell time versus conductance drop and histograms showing conductance drops. Similarly to the already reported step detection method, ${ }^{6}$ our detection method can not only detect single translocation events but also automatically distinguish levels inside every event (see the fitted signal in Supporting Information Figure 5), allowing for the calculation of the average conductance drop for each level separately rather than for the whole event. Our event and level detection method performs well even on the noisy data sets and for short-lived events and levels. In addition, it automatically categorizes events as single, two, and multilevel and discards blockage events. It then becomes possible to have histograms or any statistical characterization of a population of events with a given number of levels; this type of analysis is particularly advantageous when dealing with noisy data.

The first step in our experiments was the fabrication of nanopores. These were made on free-standing silicon nitride membranes using the focused electron beam of a transmission electron microscope (TEM) (for fabrication details see Methods section and Supporing Information Figure 1). We can make nanopores of different sizes, and for the experiments mentioned in this paper we used nanopores in the size range of 3-25 nm. After being placed in the microfluidic setup, each pore was characterized to check for linear current-voltage (IV) characteristics and low noise. We used only nanopores that have an $I_{\text {rms }}$ below $30 \mathrm{pA}$. Signals were filtered at $10 \mathrm{kHz}$ using the low-pass Bessel filter built in the Axopatch and sampled at a)

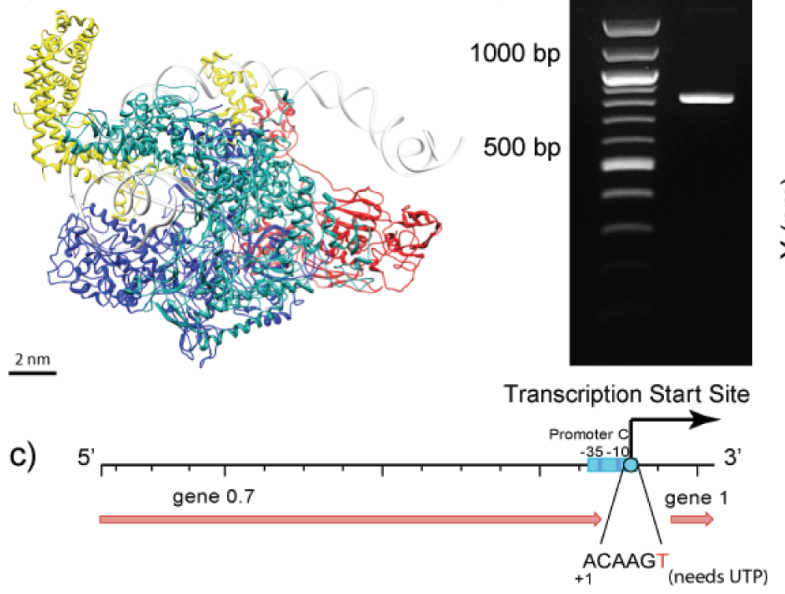

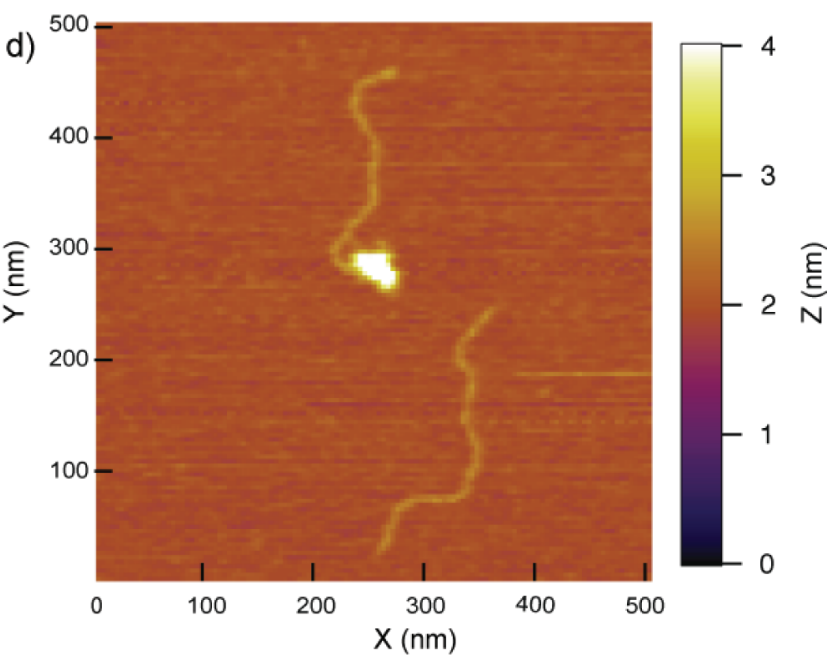

Figure 2. Schematics and AFM image of a single E. coli RNAP-DNA complex. (a) Schematics of a single E. coli RNAP-DNA complex. ${ }^{28}$ The subunits displayed are the two $\alpha$ subunits in red, $\beta$ subunit in dark blue, $\beta^{\prime}$ subunit in light blue, $\sigma$ factor in yellow, and dsDNA. (b) I: molecular weight marker, 100 bp DNA ladder; II: electrophoresis gel of $820 \mathrm{bp}$ DNA template used for nanopore assays. (c) Schematics of DNA template showing position of promoter and stall site. The E. coli RNAP promoter and transcription start site are positioned approximately at the end of the template. The stall site occurs $5 \mathrm{bp}$ downstream of the transcription start site. (d) AFM image of a single E. coli RNAP-DNA complex and a bare DNA template absorbed on mica surface. The complex is composed of an RNAP molecule, a 820 bp long DNA template, and nascent RNA (undistinguishable on the AFM image). The high and thick region corresponds to bound RNAP (height $\sim 3.90$ nm), whereas the lower and thinner more flexible lines correspond to bare DNA (height $\sim 0.46 \mathrm{~nm}$ ). 

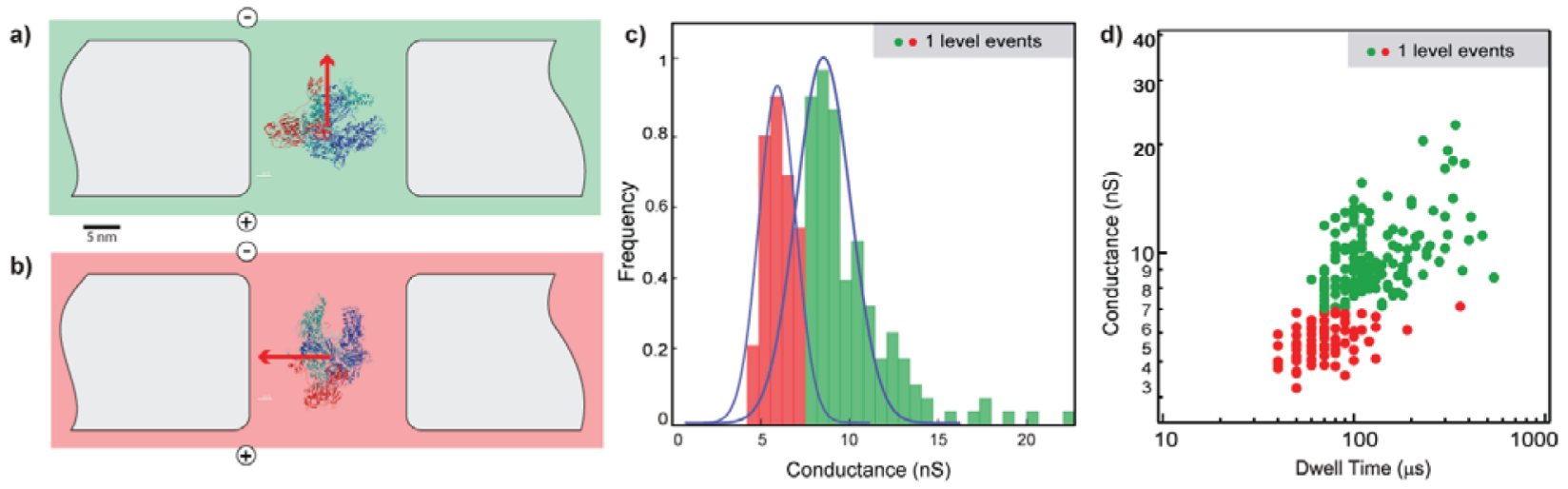

Figure 3. Translocation experiment of bare E. coli RNAP recorded at $50 \mathrm{mV}$ using a $16 \times 19 \mathrm{~nm}$ nanopore (a and b) to scale schematics of core enzyme translocating the nanopore in two different configurations. Red arrow represents the dipole moment. (a) Dipole is aligned to the electric field lines, (b) drag force is minimal, (c) conductance histogram of one-level events for E. coli RNAP at pH 7.5; two peaks are clearly resolvable. The peaks are centered at $\Delta G=5.5 \mathrm{nS}$ and $\Delta G=8.7 \mathrm{nS}$. (d) Scatter plot of one-level events separated according to the two peaks in the conductance histogram.

$100 \mathrm{kHz}$ using a National Instrument PXI-4461 DAQ card. For large nanopores, i.e., nanopores with diameters above $20 \mathrm{~nm}$, we estimated the conductance based on purely geometrical factors, approximating the actual pore to a cylinder and taking the access resistance in account according to the formula given by Hall. ${ }^{19}$ Under these approximations the conductance can be calculated with the simple expression ${ }^{31}$

$$
G=\sigma\left[\frac{4 h}{\pi d^{2}}+\frac{1}{D}\right]^{-1}
$$

Here $h$ is the length of the pore, i.e., the thickness of the $\mathrm{SiN}$ membrane $(20 \mathrm{~nm}), d$ is the average diameter of the pore $(24.5$ $\mathrm{nm})$, and $\sigma$ is the bulk conductivity of the $1 \mathrm{M} \mathrm{KCl}$ solution $\left(11.2 \mathrm{~S} \mathrm{~m}^{-1}\right) . D$ is the diameter of the pore opening, equal to $d$ in our simple approximation. This formula gives an estimated open-pore conductance of $134 \mathrm{nS}$, in good agreement with the experimental value of $114 \mathrm{nS}$.

In this study we have characterized translocation of $E$. coli RNAP, bare DNA template, and two types of E. coli RNAPDNA complex. In all reported experiments the nanopore chip was mounted in a PMMA flow cell; two silicone O-rings created a good seal between the microfluidic chambers on each side of the nanopore. Each reservoir was filled with $1 \mathrm{M} \mathrm{KCl,} 1$ $\mathrm{mM}$ Tris, and 0.1 mM EDTA buffer at $\mathrm{pH}$ 7.5. This buffer was filtered and degassed to avoid nanobubbles ${ }^{32}$ and small particles blocking the nanopore.

Our experiment started by preparing two DNA templates harboring a single promoter either toward one of its ends or in the center of the DNA template, such that we could bind and halt a single RNAP per template. To ensure that this small DNA fragment $(820 \mathrm{bp} \approx 278 \mathrm{~nm}$ ) could be detected in our nanopore setup, we first translocated it through a $3 \mathrm{~nm}$ pore; such a pore size is standard to detect short bare DNA molecules. ${ }^{3,33}$ In Supporting Information Figure $2 b, c$, we display level conductance drop histograms and scatter plots of level conductance drop versus dwell times $t_{\mathrm{D}}$ for $820 \mathrm{bp}$ DNA molecules passing through a $3 \mathrm{~nm}$ pore. For a voltage of $200 \mathrm{mV}$, we calculated a mean translocation time around 380 $\mu \mathrm{s}$, and we observed a quite broad distribution. These observation are in excellent agreement with previous studies of DNA translocation dynamics in solid-state nanopores. ${ }^{33}$ In addition, we found that the percentage of single level events was $84 \%$ versus less than $6 \%$ of real two-level events. The rest were mostly events of more than two levels. Thus, Supporting Information Figure $2 b, c$ shows that the population of events is mostly constituted of single-level events which have a peak conductance drop around $3.7 \mathrm{nS}$, stressing the fact that such a short DNA molecule can easily be detected with our setup, and the results are in good agreement with previous work done on DNA molecules inside $3 \mathrm{~nm}$ pores. ${ }^{3}$ We attempted several times unsuccessfully to detect bare 820 bp long DNA template using larger $20 \mathrm{~nm}$ pores, but due to the short duration of translocation events of bare DNA template and small current drop, we suspect that most translocation events were below the resolution of our setup. The largest pore where $820 \mathrm{bp}$ long PCR fragment could be detected was $9 \mathrm{~nm}$ (data not shown).

Next we characterized unbound E. coli RNAP. Ten units of the purchased RNAP were diluted in TE buffer. To avoid pore clogging with the buffer storing agents, we used an ultrafiltration device to exchange buffers. After buffer exchange the sample was once more diluted in $200 \mu \mathrm{L}$ of working buffer and added to the cis chamber where we applied a potential of 50 $\mathrm{mV}$ across the pore.

Among all the observed events (displayed as scatter plot in Supporting Information Figure 6) we selected only the onelevel events (69\% of the total) and plotted the level histogram (each level in an event counts for one point in the histogram) of their conductance drops in Figure $3 \mathrm{c}$ and corresponding scatter plot in Figure 3d. We observed two distinct peaks centered at $5.5 \mathrm{nS}\left(t_{\mathrm{D}} \approx 60 \mu \mathrm{s}\right)$ and $8.7 \mathrm{nS}\left(t_{\mathrm{D}} \approx 200 \mu \mathrm{s}\right)$. Our interpretation of the two peaks is illustrated in Figure $3 \mathrm{a}, \mathrm{b}$ and could be attributed to the fact that E. coli RNAP has two preferential orientations. Out of all the one-level events, $65 \%$ are attributed to the highest conductance drop (green population in Figure $3 \mathrm{~b}$ ). This conductance drop could be explained by the fact that the enzyme has a dipole ${ }^{29}$ of $5348 \mathrm{D}$ and orients itself along the electric field lines created by the potential difference between the cis and trans chambers. In that configuration this oblate ellipsoid orients its major axis perpendicular to the nanopore axis, creating a conductance drop of $8.7 \mathrm{nS}$. The rest of the one-level events (red population in Figure $3 \mathrm{~b}$ ) correspond to an RNAP molecule that would orient in order to minimize the drag force opposing the electrical force. ${ }^{34}$ The minimal dwell time for the red population is $40 \mu \mathrm{s}$, whereas the minimal dwell time for the 
a)
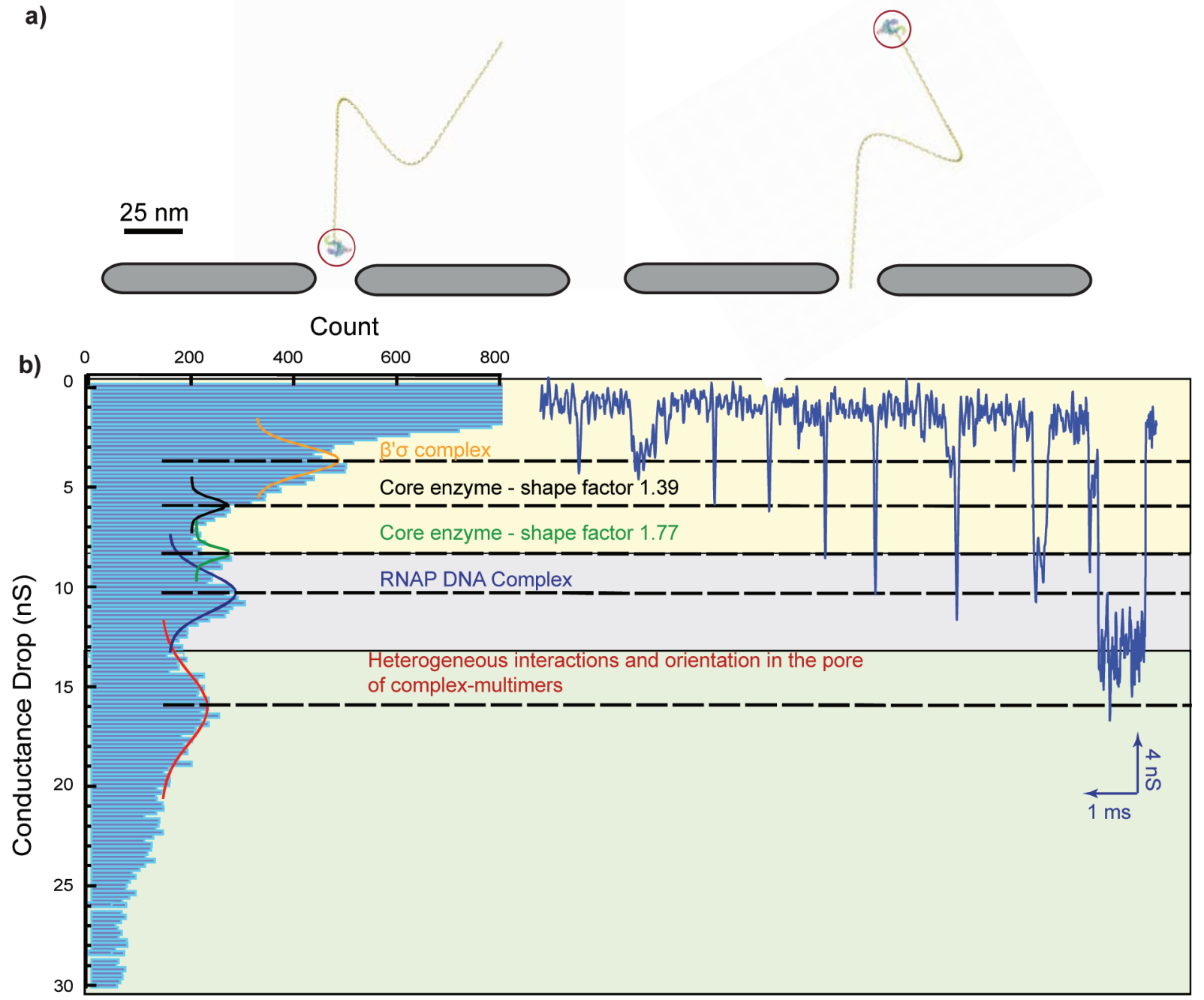

Figure 4. Translocation experiment of E. coli RNAP-DNA complex recorded at $50 \mathrm{mV}$ using a $16 \times 19 \mathrm{~nm}$ nanopore (a) to scale schematics of possible translocation for RNAP-DNA complex. (b) Point histogram where main peaks are fitted. The peak centered at $\Delta G=4 \mathrm{nS}$ has been attributed to the translocation of the $\beta^{\prime} \sigma$ complex, whereas the second and third peaks centered at $\Delta G=6.3$ and $8.8 \mathrm{nS}$ have been attributed to the translocation of the core enzyme with different orientations. The peak centered at $\Delta G=10.6 \mathrm{nS}$ has been attributed to the translocation of RNAPDNA complex, while the last fitted peak could be due to the heterogeneous interactions and orientations of multimers inside the pore.

green population is $70 \mu \mathrm{s}$. This observation comforts our interpretation that when the RNAP molecule orients itself in the configuration shown in Figure 3a, it has to counter more drag forces and so it translocates slower.

Moreover, the volume excluded by the $\mathrm{RNAP}^{35}$ is much smaller compared to the nanopore volume accounting for the small conductance drops. As we fabricated our nanopores using the standard electron beam drilling method, nanopores geometry should be a double cone ${ }^{36}$ where the $h_{\text {eff }}=18 \mathrm{~nm}$ and the average diameter is $17.5 \mathrm{~nm}$, which gives a volume of $4300 \mathrm{~nm}^{3}$ for this nanopore. While using Gerstein's calcvolume program, ${ }^{37}$ we estimated the E. coli RNAP volume to be $600 \mathrm{~nm}^{3}$.

To estimate conductance drops, we have considered a concept of excluded volumes proposed in previous reports 35,38 where the conductance drop is proportional to the volume excluded by the molecule that passes through the nanopore.
The predicted conductance drop can be calculated using the equation

$$
\Delta G=\gamma \sigma \frac{V_{\text {excluded }}}{H_{\mathrm{eq}}{ }^{2}} f\left(\frac{d_{\mathrm{p}}}{d_{\mathrm{m}}}\right)
$$

where $\gamma$ is a shape factor, $\sigma$ the solution conductivity, $V_{\text {excluded }}$ the volume excluded by the molecule that translocates the nanopore, $H_{\mathrm{eq}}$ the equivalent nanopore thickness, and $f$ a correction factor that depends on relative pore/molecule geometry. For $H_{\text {eq }}$ we have used the formula ${ }^{39}$

$$
H_{\mathrm{eq}}=h_{\mathrm{p}}+0.8 d_{\mathrm{p}}
$$

with $h_{\mathrm{p}}$ the membrane thickness and $d_{\mathrm{p}}$ the pore diameter.

For $V_{\text {excluded }}$ we have used the atomic volume of the molecule calculated using Gerstein's calc-volume program. ${ }^{37}$ As it is done in other studies, ${ }^{35,38}$ the $f$ factor is considered equal to one as a first approximation. For an equivalent nanopore thickness of 34 $\mathrm{nm}$, a conductance of $11.2 \mathrm{~S} \mathrm{~m}^{-1}$, an excluded volume of 518 $\mathrm{nm}^{3}$ (core enzyme), and a shape factor of 1.39 (see Supporting Information Appendix for calculations), we obtain a predicted 
conductance drop of $7.0 \mathrm{nS}$. The value observed in our experiments is $5.5 \mathrm{nS}$. Similarly, we obtain a predicted conductance drop of $8.9 \mathrm{nS}$ for a shape factor of 1.77 , and the observed value is $8.7 \mathrm{nS}$. Many factors such as true pore size, estimated hydrodynamic diameter of the translocating molecules, ionic fluctuations inside the pore, and signal filtering could contribute to the disagreement between theoretical and observed values of conductance drops.

Having characterized the behavior of bare DNA molecules and unbound $E$. coli RNAP, we proceeded by preparing E. coli RNAP-DNA complexes. We performed a transcription reaction in a standard transcription buffer as recommended by the manufacturer but lacking one nucleotide so that the $E$. coli RNA polymerase stalled on the DNA template when having to incorporate this nucleotide, i.e., five bases downstream of the transcription start site in our template (Figure 2c). We then performed ultrafiltration to remove free nucleotides and to exchange the transcription buffer to the deposition buffer used for atomic force microscope (AFM) imaging. An aliquot was then incubated on a mica disk and imaged under an AFM (Figure 2d). We observed a mixture of DNA fragments complexed with the RNA polymerase, bare DNA fragments, and free RNAP (not seen in the image field in Figure 2d). Consistent with the location of the promoter sequence close to one end of the fragment, we observed RNAP at one and only one end of the DNA molecule. This important verification step indicated that the promoter was functional and we could form E. coli RNAP-DNA complexes.

To check whether the RNAP-DNA complex was resistant to high salt conditions, required in nanopore sensing experiments, we repeated the AFM imaging after incubating the sample for $30 \mathrm{~min}$ in $1 \mathrm{M} \mathrm{KCl}$. A total surface of $250 \mu \mathrm{m}^{2}$ was scanned at high resolution on both samples. Out of around 150 molecules counted, we observed $63.8 \%$ of RNAP-DNA complex versus $36.2 \%$ of bare DNA molecules in $1 \mathrm{M} \mathrm{KCl}$, compared to $70.2 \%$ and $29.8 \%$ of complexes and bare DNA molecules, respectively, in regular buffer conditions. Thus, the AFM data indicated that we obtained a mixture of bare template DNA molecule, free RNAP, and RNAP-DNA complexes that was stable under high salt conditions (Supporting Information Figure 3).

To study translocation of this RNAP-DNA complex where RNAP is at one end of the template, we chose a pore size of 16 $\times 19 \mathrm{~nm}$, slightly larger than the diameter of the RNAP measured on the EM structure shown in Figure 2a. On the one hand, selected pore size is large enough to allow the translocations of complexes. On the other hand, it is small enough to promote interactions of the complexes with the pore walls, as already shown previously for DNA molecules in small pores where interactions with the wall reduce the DNA velocity. ${ }^{33}$ We diluted the sample into $1 \mathrm{M} \mathrm{KCl}$, a salt concentration that is crucial for high SNR ratio in nanopore sensing experiments, and added the mixture to the cis chamber of our microfluidic setup. We applied a voltage of $50 \mathrm{mV}$ across the pore and shortly after we started seeing events.

We noticed that the base current was occasionally drastically reduced for a couple of seconds (up to $5 \mathrm{~s}$ ) but then returned to its original value. This phenomenon was probably due to a complex being caught in the nanopore (see Supporting Information Figure 4): we termed these events "blockages" and discarded them from further analysis, keeping only the shorter events, which were processed as described in the Methods section. The sample contained a mixture of bare DNA template, free RNAP, $\beta^{\prime} \sigma$ complexes, and RNAP-DNA complexes. The $\beta^{\prime} \sigma$ complex is native and stable in $6 \mathrm{M}$ urea and requires the addition of $20 \%$ formamide for dissociation. ${ }^{40}$

Among the many possible translocation events this sample can produce, we assume that the RNAP-DNA complex can either pass through the pore with DNA going in first or RNAP going in first and DNA follows. Those two configurations are illustrated to scale Figure 4a. At a voltage of $50 \mathrm{mV}$ the noise level is low enough to separate the different peaks in a point histogram (where each count is a point from the event), which we use to identify the major conductance drops.

The 820 bp DNA fragment $(\approx 278 \mathrm{~nm}$, below $3 \mathrm{Kuhn}$ lengths) is unlikely to translocate in a folded conformation. The size of this DNA fragment was selected to minimize the occurrence of folded DNA molecule translocations. ${ }^{6,41}$ To estimate the conductance drops for folded and unfolded DNA, we use the well-studied $\lambda$ DNA. Such an experiment allowed us to evaluate the incidence of two-level events resulting from translocation of the folded DNA state and the performance of our code in terms of classifying events.

We translocated $\lambda$ DNA through a pore of average diameter $21 \mathrm{~nm}$ (see Supporting Information Figure 2d), similar size as the pore used to study the RNAP-DNA complex. The corresponding scatter plot and level conductance histogram are displayed in Supporting Information Figure 2e,f. For a voltage of $200 \mathrm{mV}$, the level conductance drops were centered at $\Delta G=$ $1 \pm 0.2 \mathrm{nS}$. By closely inspecting only two-level events that have been previously attributed to the translocation of the partially folded $\mathrm{DNA},{ }^{3}$ we can conclude that the measured conductance drop for unfolded DNA in a $21 \mathrm{~nm}$ pore is $1 \mathrm{nS}$ while for folded DNA it is $2 \mathrm{nS}$. A typical two-level event is displayed in the inset of Supporting Information Figure $2 \mathrm{f}$. These values are below the values of 4-16 nS observed in experiments with E. coli RNAP-DNA complex, which indicates that those drops are not due to DNA.

From the histogram Figure $4 \mathrm{~b}$ we fit the main peaks and interpret them using the same calculations as before. Since this experiment is done in the same nanopore and at the same voltage as the experiment illustrated in Figure 3, the predicted conductance drops induced by free RNAP translocating the pore are the same. The observed conductance drops for a free RNAP with a shape factor of 1.39 is $6.3 \mathrm{nS}$ and the one for a shape factor of 1.77 is $8.8 \mathrm{nS}$, which fits nicely with the predicted conductance drops of 7.0 and $8.9 \mathrm{nS}$, respectively. For the atomic volume of the $\beta^{\prime} \sigma$ complex, $307 \mathrm{~nm}^{3}$, using a shape factor of 1.36 (see Supporting Information Appendix for details), we find a conductance drop of $4.1 \mathrm{nS}$, which fits perfectly the observed conductance drop of $4.0 \mathrm{nS}$. For the volume calculation of the RNAP-DNA complex we consider only the volume of the complex that is in $H_{\mathrm{eq}}$ (see Supporting Information Appendix). Since the equivalent height of this cylinder is calculated using eq 3 and since we suppose that the DNA is mostly straight within that region, we consider an excluded volume of 598 (holoenzyme) $+72\left(\right.$ DNA in $\left.H_{\text {eq }}\right)=$ $670 \mathrm{~nm}^{3}$ for the complex. We obtain a predicted conductance drop of $9.7 \mathrm{nS}$ in comparison to the observed value of $10.6 \mathrm{nS}$. Our results demonstrate that it is possible to distinguish $\beta^{\prime} \sigma$ complex, core enzyme, and E. coli RNAP-DNA complexes.

The events that are around $16.0 \mathrm{nS}$ fitted by the red curve represent $24 \%$ of the total number of events, and most of them are multilevel events and longer in dwell times. Long and deep events will result in many more points when displayed in the form of a point histogram compared to a level histogram where they result in one point (see Supporting Information Figure 6). 
a)

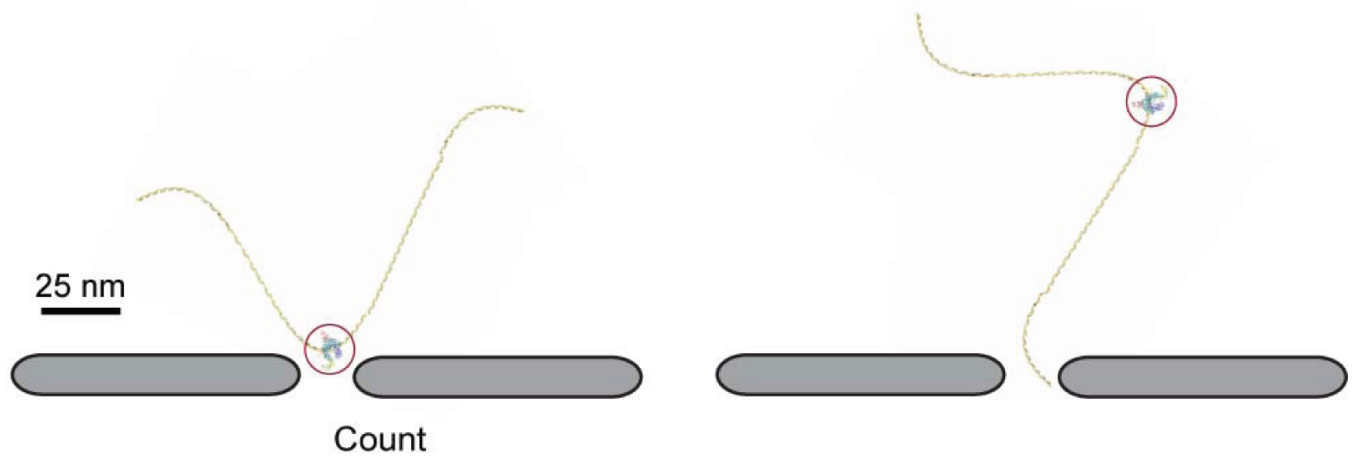

b)

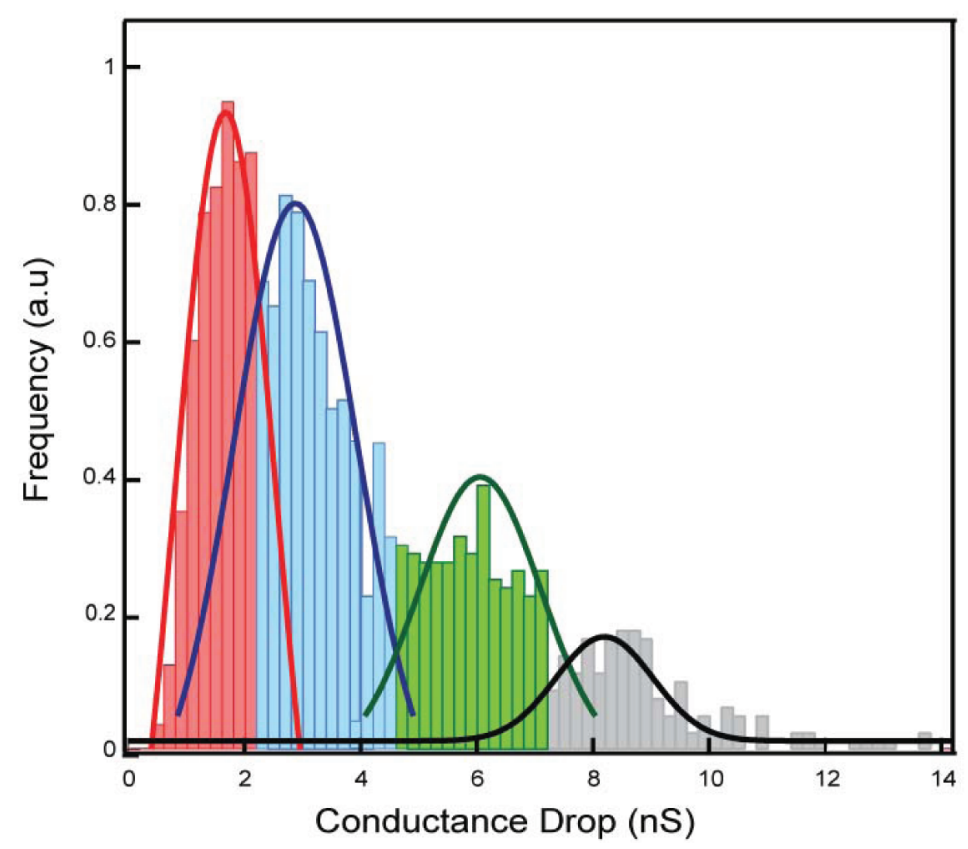

Figure 5. Translocation experiment of E. coli RNAP-DNA complex recorded at $200 \mathrm{mV}$ using a $20 \times 29 \mathrm{~nm}$ nanopore (a) to scale schematics of possible translocation for RNAP-DNA complex. (b) Level histogram of multilevel events where main peaks are fitted. The peak centered at $\Delta G=$ $1.7 \mathrm{nS}$ has been attributed to the translocation of the $\beta$ or $\beta^{\prime}$ subunit that is separated from the core enzyme due to high applied voltage, while the second and third peaks centered at $\Delta G=3.1$ and $4.2 \mathrm{nS}$ have been attributed to the translocation of the $\beta^{\prime} \sigma$ complex with different orientations and last peak centered at $\Delta G=8.2 \mathrm{nS}$ has been attributed to the RNAP-DNA complex.

More complex orientation and pore interaction mechanisms of multimers or their compression could explain the origin of those long and deep events. Such events are mostly observed in this experiment at low voltage, thus comforting the idea that low voltages cannot exert enough force to separate multimers.

In the last experiment, RNAP-DNA complexes with RNAP halted in the center of the DNA template are translocated (see illustration in Figure 5a) through a $20 \times 29 \mathrm{~nm}$ pore. As previously, the sample contains a mixture of bare DNA, free RNAP translocating in two preferential orientations, $\beta^{\prime} \sigma$ complex, and the RNAP-DNA complex. Figure $5 \mathrm{~b}$ shows a level histogram of multilevel events only. This experiment was done at a higher voltage $(200 \mathrm{mV})$ and in a bigger pore $(20 \times$ $29 \mathrm{~nm})$; thus $H_{\mathrm{eq}}$ was bigger $(39.6 \mathrm{~nm})$. Experimental conditions, mainly applied voltage, implied that the noise was higher than in previous experiments and caused harder identification of the peaks; therefore, we chose to display conductance drops using level histograms.

Four major conductance drops can be fitted from this histogram and are displayed with fits in Figure $5 \mathrm{~b}$. In the same way we fit conductance drops using eq 2 . The smallest conductance drop is attributed to the $\beta$ or $\beta^{\prime}$ subunit (of similar volume) that could have detached from the core enzyme because of high voltage, ${ }^{35}$ confirming that high voltages could exert enough force to separate subunits. $\beta^{\prime} \sigma$ complex is also observed in two preferential configurations. Like the core enzyme, $\beta^{\prime} \sigma$ has a dipole of $4852 \mathrm{D}$ and is ellipsoidal. The observed conductance drops for both configurations are 3.1 and $4.2 \mathrm{nS}$, and the theoretical drops are 3.0 and $4.1 \mathrm{nS}$, respectively. We also observe the core enzyme with a predicted conductance drop of $5.6 \mathrm{nS}$ and an observed conductance drop of $6.0 \mathrm{nS}$. For the complex, we did the same reasoning and found an excluded volume of 598 (holoenzyme) +144 (DNA) $=742 \mathrm{~nm}^{3}$, which predicts a conductance drop of $8.0 \mathrm{nS}$. Once again the observed value is $8.2 \mathrm{nS}$, which fits well with our interpretation.

In conclusion, we have shown that single E. coli RNAP and $E$. coli RNAP-DNA transcription complexes can be detected using nanopore sensing. This technique has a resolution capable of detecting a single protein on a DNA molecule and their orientation during pore translocation. Large net charge carried by E. coli RNAP together with its ellipsoid shape and its 
electric dipole might be responsible for enzyme orientations that resulted in observed conductance drops. With sufficient voltage, we demonstrated that the RNAP subunits could be separated from the core enzyme. ${ }^{35}$ Our measurements allowed us to identify those different subunits. The ability to tailor solidstate nanopores to any desired size down to $1 \mathrm{~nm}$ opens up possibilities to study a very broad range of protein-DNA complexes under various experimental conditions. Nanopore sensing is thus a versatile technique, which could be complemented with other single-molecule modalities ${ }^{42-44}$ to allow for detailed description of biological processes at the single molecule level.

Methods. Nanopore Fabrication and Experimental Conditions. Nanopore chips were fabricated using standard cleanroom processes. The starting substrate was a double-sided polished silicon wafer with a triple stack of insulating layers: 20 nm of low stress $\mathrm{Si}_{3} \mathrm{~N}_{4}, 100 \mathrm{~nm}$ of $\mathrm{SiO}_{2}, 100 \mathrm{~nm}$ low stress $\mathrm{Si}_{3} \mathrm{~N}_{4}$. The supporting silicon and insulating layers on the back side were dry and wet etched to create a $50 \mu \mathrm{m}^{2}$ window that formed a thick membrane. Using electron beam lithography followed by reactive ion etching, we defined a $20 \mathrm{~nm}$ thick 500 $\mathrm{nm}^{2}$ region. Nanopores from 3 to $20 \mathrm{~nm}$ were drilled in the center of this thinned region with a Philips/FEI CM300 TEM operated at $200 \mathrm{kV}$ using spot size 5 (see Supporting Information Figure 1). After fabrication the pores were stored in a degassed and filtered 1:1 $\mathrm{ddH}_{2} \mathrm{O}: \mathrm{EtOH}$ solution until use. $^{43}$

The nanopore chip was mounted in a custom-designed microfluidic cell, and the two reservoirs on each side of the pore were filled with 0.22 um filtered and degassed buffer containing $1 \mathrm{M} \mathrm{KCl}, 1 \mathrm{mM}$ Tris/ $\mathrm{HCl} \mathrm{pH} 7.5$, and $0.1 \mathrm{mM}$ EDTA. An Axopatch 200B (Molecular Devices, Inc., Sunnyvale, CA) was used to amplify the ionic current through the nanopore. The $\mathrm{Ag} / \mathrm{AgCl}$ electrodes were immersed in the reservoirs on each side of the nanopore and connected to the Axopatch preamplifier. This part of the setup was mounted on a damping breadboard (Thorlabs, NJ) and encapsulated in a Faraday cage. The RNAP-DNA complex was introduced into the cis side of the nanopore, and a voltage of $50 \mathrm{mV}$ was applied to the trans side. For experiments with bare DNA template, a voltage of 200 and $300 \mathrm{mV}$ was applied.

Sample Preparation: RNA Polymerase-DNA Complex. To engineer a DNA template onto which we could bind and then pause E. coli RNA polymerase, we chose an $820 \mathrm{bp}$ fragment containing the $\mathrm{C}$ promoter of $\mathrm{T} 7$ bacteriophage DNA (GeneON, Germany). In this fragment, the promoter spans a region between positions 661 and 723 . The transcription start site is at position 709 . The enzyme $100 \%$ saturated with sigma factor, which is required for specific initiation of RNA synthesis bacterial or phage E. coli RNA polymerase promoters, was obtained from (Affymetrix, CA). The promoter sequence was 5'-ATTGATAAGC AACTTGACGC AATGTTAATG GGCTGATAGT CTTATCTTAC AGGTCATCTG CGG-3', with the $-\overline{35 \text { and }}-10$ sequence of the $C$ promoter underlined. The primers used to amplify this DNA fragment from $\mathrm{T} 7$ bacteriophage DNA were 5'-GGATGCTATACGGTGG'TACTTG-3' and 5'-GAGACTGTAGCTTGACCGACGA-3'.

A similar template was engineered so that the RNA polymerase would bind and then pause in the center of an $806 \mathrm{bp}$ DNA fragment. The primers used to amplify this DNA fragment were 5'-ATCGACCCTGAGGAACTCATC-3' and 5'CTTCCGGCTTGATTTCTTGC-3'.
Using PCR, we amplified the DNA fragments and analyzed it by gel electrophoresis ( $1 \%$ agarose gel stained with $\mathrm{EtBr}$ prior to imaging). We could see a band that corresponded to the expected size of DNA (see Figure 2). The product was then purified with a PCR cleanup kit (Macherey-Nagel) and used to prepare the RNAP-DNA complex. To prepare this complex, we performed an in vitro transcription reaction in a total volume of $500 \mu \mathrm{L}$ containing $40 \mathrm{mM}$ Tris- $\mathrm{HCl}, \mathrm{pH} 7.5,150$ $\mathrm{mM} \mathrm{KCl}, 10 \mathrm{mM} \mathrm{MgCl}_{2}, 0.01 \%$ Triton X-100, $10 \mathrm{mM} \mathrm{DTT}$, $50 \mu \mathrm{L}$ of NTP mix (10 mM ATP, $10 \mathrm{mM} \mathrm{GTP,} 10 \mathrm{mM} \mathrm{CTP})$, $6 \mu \mathrm{g}$ of DNA fragment, and 300 units of E. coli RNA polymerase. The mixture was incubated for $10 \mathrm{~min}$ at $37^{\circ} \mathrm{C}$ to allow the polymerase to form an open complex, start transcription, and stall after transcribing 5 nucleotides because of the lack of UTP in the reaction. The mixture was then subjected to ultrafiltration (Pierce Concentrators, $150 \mathrm{kDa}$ filter from Thermo Scientific) to clean the reaction from detergents and remove free nucleotides.

AFM. For AFM imaging, part of the concentrated DNAprotein complex was diluted to $1 \mathrm{ng} / \mu \mathrm{L}$ of $\mathrm{DNA}$ in the deposition buffer: $5 \mathrm{mM}$ Tris/ $\mathrm{HCl} \mathrm{pH} 8,10 \mathrm{mM} \mathrm{MgCl}_{2}$. It was then incubated on freshly cleaved mica for $2 \mathrm{~min}$ and rinsed thoroughly with $\mathrm{ddH}_{2} \mathrm{O}$. All AFM images were acquired in air and in tapping mode with an Asylum Research Cypher microscope. We used Olympus silicon cantilevers (OMCLAC160TS- Olympus) with force constants of 1.9-2.3 N/m. Images were recorded with typical scan rates of $1.0-2.0 \mathrm{~Hz}$.

Data Acquisition. Signals were filtered at $10 \mathrm{kHz}$ using the low-pass Bessel filter built-in the Axopatch and sampled at 100 $\mathrm{kHz}$ using a National Instrument PXI-4461 DAQ card. This allowed for low-noise measurement. To allow for discrimination of sharp spikes (termed events) above the noise level, a custom-made LabVIEW program only recorded data when the current signal peak depth was above $4 \times I_{\text {rms }}$, and the event was longer than $50 \mu \mathrm{s}$, which made files lighter and easier to analyze. This data signal was then fitted with an algorithm ${ }^{45}$ that detects abrupt rupture changes in a short time estimate of the averaged signal (see for example signal in Supporting Information Figure 5a). Data analysis was performed with MATLAB software. The algorithm for event detection needed to be robust enough to detect rupture points-start and stop of the events-but also to find the number of levels in an event in order to determine whether it was bare DNA template translocating through the nanopore or if it was the RNAPDNA complex. Finally, event dwell times were computed and conductance drops of the different levels inside each event were calculated and classified as one, two, or multilevel event. All histograms were computed based on conductance drops; those were calculated by subtracting the fitted value of the surrounding base conductance to the mean value of each level inside each event.

\section{ASSOCIATED CONTENT}

\section{Supporting Information}

Six additional Figures and Appendix. This material is available free of charge via the Internet at http://pubs.acs.org.

\section{AUTHOR INFORMATION}

\section{Corresponding Author}

*E-mail aleksandra.radenovic@epfl.ch.

\section{Notes}

The authors declare no competing financial interest. 


\section{ACKNOWLEDGMENTS}

This work was financially supported by European Research Council (grant no. 259398, PorABEL: Nanopore integrated nanoelectrodes for biomolecular manipulation and sensing). C.R. was financed by a grant from the Swiss SystemsX.ch initiative (IPhD), evaluated by the Swiss National Science Foundation. F.T. was partially financed by FP7 nanoDNA sequencing grant. We thank the Centre Interdisciplinaire de Microscopic Electronique (CIME) at EPFL for access to electron microscopes. Special thank to DTL Alexander for providing training and technical assistance with the transmission electron microscope. We also thank P. Granjon (GIPSA-lab, INPG) for providing the detection change software module that is part of his lecture. Nanopore fabrication was carried out in part in at the EPFL Center for Micro/Nanotechnology (CMI). Thanks to K. Lister (CMI) for technical support with electron-beam lithography. We thank A. Kis for the use of AFM.

\section{REFERENCES}

(1) Li, J.; Stein, D.; McMullan, C.; Branton, D.; Aziz, M. J.; Golovchenko, J. A. Nature 2001, 412 (6843), 166-169.

(2) Fologea, D.; Gershow, M.; Ledden, B.; McNabb, D. S.; Golovchenko, J. A.; Li, J. L. Nano Lett. 2005, 5 (10), 1905-1909.

(3) Li, J. L.; Gershow, M.; Stein, D.; Brandin, E.; Golovchenko, J. A. Nat. Mater. 2003, 2 (9), 611-615.

(4) Skinner, G. M.; van den Hout, M.; Broekmans, O.; Dekker, C.; Dekker, N. H. Nano Lett. 2009, 9 (8), 2953-2960.

(5) Smeets, R. M. M.; Keyser, U. F.; Krapf, D.; Wu, M. Y.; Dekker, N. H.; Dekker, C. Nano Lett. 2006, 6 (1), 89-95.

(6) Storm, A. J.; Chen, J. H.; Zandbergen, H. W.; Dekker, C. Phys. Rev. E 2005, 71, 051903.

(7) van den Hout, M.; Krudde, V.; Janssen, X. J. A.; Dekker, N. H. Biophys. J. 2010, 99 (11), 3840-3848.

(8) Wanunu, M.; Dadosh, T.; Ray, V.; Jin, J. M.; McReynolds, L.; Drndic, M. Nat. Nanotechnol. 2010, 5 (11), 807-814.

(9) Heins, E. A.; Siwy, Z. S.; Baker, L. A.; Martin, C. R. Nano Lett. 2005, 5 (9), 1824-1829.

(10) Kowalczyk, S. W.; Hall, A. R.; Dekker, C. Nano Lett. 2010, 10 (1), 324-8.

(11) Stefureac, R. I.; Trivedi, D.; Marziali, A.; Lee, J. S. J. Phys.: Condens. Matter 2010, 22 (45), 454133.

(12) Pastoriza-Gallego, M.; Rabah, L.; Gibrat, G.; Thiebot, B.; Goot, F. G.; Auvray, L.; Betton, J. M.; Pelta, J. J. Am. Chem. Soc. 2011, 133 (9), 2923-2931.

(13) Smeets, R. M. M.; Kowalczyk, S. W.; Hall, A. R.; Dekker, N. H.; Dekker, C. Nano Lett. 2009, 9 (9), 3089-3095.

(14) Sischka, A.; Spiering, A.; Khaksar, M.; Laxa, M.; Konig, J.; Dietz, K. J.; Anselmetti, D. J. Phys.: Condens. Matter 2010, 22 (45), 454121.

(15) Dorvel, B.; Sigalov, G.; Zhao, Q.; Comer, J.; Dimitrov, V.; Mirsaidov, U.; Aksimentiev, A.; Timp, G. Nucleic Acids Res. 2009, 37 (12), 4170-4179.

(16) Wanunu, M.; Sutin, J.; Meller, A. Nano Lett. 2009, 9 (10), 3498-3502.

(17) Singer, A.; Wanunu, M.; Morrison, W.; Kuhn, H.; FrankKamenetskii, M.; Meller, A. Nano Lett. 2010, 10 (2), 738-742.

(18) Wanunu, M.; Cohen-Karni, D.; Johnson, R. R.; Fields, L.; Benner, J.; Peterman, N.; Zheng, Y.; Klein, M. L.; Drndic, M. J. Am. Chem. Soc. 2011, 133 (3), 486-492.

(19) Hall, A. R.; van Dorp, S.; Lemay, S. G.; Dekker, C. Nano Lett. 2009, 9 (12), 4441-4445.

(20) Kowalczyk, S. W.; Kapinos, L.; Blosser, T. R.; Magalhaes, T.; van Nies, P.; Lim, R. Y.; Dekker, C. Nat. Nanotechnol. 2011, 6 (7), 433-8.

(21) Benner, S.; Chen, R. J. A.; Wilson, N. A.; Abu-Shumays, R.; Hurt, N.; Lieberman, K. R.; Deamer, D. W.; Dunbar, W. B.; Akeson, M. Nat. Nanotechnol. 2007, 2 (11), 718-724.

(22) Alberts, B. Cell 1998, 92 (3), 291-294.
(23) Bustamante, C.; Cheng, W.; Meija, Y. X. Cell 2011, 144 (4), $480-497$.

(24) Neuman, K. C.; Abbondanzieri, E. A.; Landick, R.; Gelles, J.; Block, S. M. Cell 2003, 115 (4), 437-447.

(25) Shaevitz, J. W.; Abbondanzieri, E. A.; Landick, R.; Block, S. M. Nature 2003, 426 (6967), 684-7.

(26) Galburt, E. A.; Grill, S. W.; Wiedmann, A.; Lubkowska, L.; Choy, J.; Nogales, E.; Kashlev, M.; Bustamante, C. Nature 2007, 446 (7137), 820-3.

(27) Margeat, E.; Kapanidis, A. N.; Tinnefeld, P.; Wang, Y.; Mukhopadhyay, J.; Ebright, R. H.; Weiss, S. Biophys. J. 2006, 90 (4), 1419-1431.

(28) Lawson, C. L.; Hudson, B. P.; Quispe, J.; Lara-Gonzalez, S.; Kim, Y.; Berman, H. M.; Arnold, E.; Ebright, R. H. Proc. Natl. Acad. Sci. U. S. A. 2009, 106 (47), 19830-19835.

(29) Felder, C. E.; Prilusky, J.; Silman, I.; Sussman, J. L. Nucleic Acids Res. 2007, 35, W512-521.

(30) Manning, G. S. J. Chem. Phys. 1969, 51 (3), 924-1024.

(31) Kowalczyk, S. W.; Grosberg, A. Y.; Rabin, Y.; Dekker, C. Nanotechnology 2011, 22 (31), 315101.

(32) Smeets, R. M. M.; Keyser, U. F.; Wu, M. Y.; Dekker, N. H.; Dekker, C. Phys. Rev. Lett. 2006, 97 (8), 088101.

(33) Wanunu, M.; Sutin, J.; McNally, B.; Chow, A.; Meller, A. Biophys. J. 2008, 95 (10), 4716-4725.

(34) van Dorp, S.; Keyser, U. F.; Dekker, N. H.; Dekker, C.; Lemay, S. G. Nat. Phys. 2009, 5 (5), 347-351.

(35) Talaga, D. S.; Li, J. J. Am. Chem. Soc. 2009, 131 (26), 9287-97.

(36) Kim, M. J.; Wanunu, M.; Bell, D. C.; Meller, A. Adv. Mater. 2006, 18 (23), 3149-3153.

(37) Voss, N. R.; Gerstein, M. J. Mol. Biol. 2005, 346 (2), 477-492.

(38) Yusko, E. C.; Johnson, J. M.; Majd, S.; Prangkio, P.; Rollings, R. C.; Li, J. L.; Yang, J.; Mayer, M. Nat. Nanotechnol. 2011, 6 (4), 253260.

(39) Grover, N. B.; Naaman, J.; Bensasso., S; Doljansk., F.; Nadav, E. Biophys. J. 1969, 9 (11), 1415-1425.

(40) Zillig, W.; Palm, P.; Heil, A. Function and reassembly of subunits of DNA-dependent RNA polymerase. In RNA Polymerase; Losick, R., Chamberlin, M., Eds.; Cold Spring Harbor Laboratory Press: Cold Spring Harbor, NY, 1976; Vol. 6, pp 101-125.

(41) Kotsev, S.; Kolomeisky, A. B. J. Chem. Phys. 2007, 127 (18), 185103.

(42) Keyser, U. F.; Koeleman, B. N.; Van Dorp, S.; Krapf, D.; Smeets, R. M. M.; Lemay, S. G.; Dekker, N. H.; Dekker, C. Nat. Phys. 2006, 2 (7), 473-477.

(43) Trepagnier, E. H.; Radenovic, A.; Sivak, D.; Geissler, P.; Liphardt, J. Nano Lett. 2007, 7 (9), 2824-2830.

(44) Spiering, A.; Getfert, S.; Sischka, A.; Reimann, P.; Anselmetti, D. Nano Lett. 2011, 11 (7), 2978-2982.

(45) Basseville, M.; Nikiforov, I. G. Detection of Abrupt Changes: Theory and Application; Prentice-Hall: Englewood Cliffs, NJ, 1993. 